\title{
ERRATUM: COMPLEMENTS AND HIGHER RESONANCE VARIETIES OF HYPERPLANE ARRANGEMENTS
}

\author{
Nero Budur
}

\begin{abstract}
This is an Erratum to Complements and higher resonance varieties of hyperplane arrangements, Math. Res. Lett. 18(5) (2011), 859-873.
\end{abstract}

The proof of $[1$, Theorem 1.1, p. 867] is incomplete: for the claimed equality of two sets, only one of the inclusions is proved. It was assumed wrongly that the other inclusion is easy. In fact, the other inclusion holds only under an additional hypothesis. We correct here Theorem 1.1 and Corollary 1.2 of [1]. The rest of the results of [1] stay the same, including the main results of the paper on deeper propagation and dimensional bounds for resonance varieties and the positivity results on Betti numbers.

Theorem 1.1. Let $i \leq n-2$. (a) $\mathbb{P}\left(R_{1}^{i}(U)\right)$ is the support of the ideal $\mathcal{I}_{\beta_{i}}\left(\phi_{i}\right)$. (b) $\mathbb{P}\left(R_{j}^{i}(U)\right)$ contains the support of the ideal $\mathcal{I}_{\beta_{i}+1-j}\left(\phi_{i}\right)$, and equals it away from $\mathbb{P}\left(R_{1}^{i-1}(U)\right)$. (c) $\mathbb{P}\left(R_{j}^{1}(U)\right)$ is the support of the ideal $\mathcal{I}_{\beta_{1}+1-j}\left(\phi_{1}\right)$. (d) $\mathbb{P}\left(R_{2}^{i}(U)\right)$ is the support of the ideal $\mathcal{I}_{\beta_{2}+1-j}\left(\phi_{2}\right)$. (e) $\mathbb{P}\left(R_{j}^{i}(U)\right)$ is the support of the ideal $\mathcal{I}_{\beta_{i}+1-j}\left(\phi_{i}\right)$ if $j \leq 1+(n-3) / i$. (f) $\mathbb{P}\left(R_{j}^{i}(U)\right)$ is the support of the ideal $\mathcal{I}_{b_{i}+1-j}\left(\phi_{i-1} \oplus \phi_{i}\right)$.

Proof. Part (a) is [1, Proposition 3.4]. The first claim of (b) is what is actually proven on p. 867 of [1]. Indeed, it follows from: if $(R, P)$ is a local Noetherian domain and $M$ is an $R$-module minimally generated by $k$ or more elements, then $k-\operatorname{rank}(M) \leq \operatorname{dim} \operatorname{Tor}_{1}^{R}(R / P, M)$. The proof of this statement involves the obvious fact that $\operatorname{rank}(N) \leq k^{\prime}$ where $N$ are the first syzygies of $M$ and $k^{\prime}$ is the minimal number of generators of $N$ (note that there is a typo in loc. cit.). The converse of the statement holds if and only if $N$ is free. Since $N$ and $M$ are the stalks of $\operatorname{coker}\left(\phi_{i-1}\right)$ and $\operatorname{coker}\left(\phi_{i}\right)$ in our case, by part (a) this gives the second claim in (b). Since $\mathbb{P}\left(R_{1}^{0}(U)\right)=\emptyset$, (c) follows. Budur [1, Corollary 1.3] holds as stated due to the right inclusion in (b) and it follows from the same statements about the corresponding determinantal ideals. Thus, $\mathbb{P}\left(R_{1}^{i-1}(U)\right)$ is a subset of the support of $\mathcal{I}_{\beta_{2}+1-j}\left(\phi_{2}\right)$, and (d) follows by (b). Similarly, (e) follows from the second part of [1, Corollary 1.3] for determinantal ideals. Part (f) is well-known.

We do not know if the original statement of Theorem 1.1, that $\mathbb{P}\left(R_{j}^{i}(U)\right)$ is the support of $\mathcal{I}_{\beta_{i}+1-j}\left(\phi_{i}\right)$, holds in general. Due to the right inclusion of part (b) in Theorem 1.1 above, this change affects in [1] only the statement of Corollary 1.2:

Corollary 1.2. Let $i \leq n-2$. If $\left(\beta_{i-1}+j\right)\left(\beta_{i+1}+j\right)<d-2$, then $\mathbb{P}\left(R_{j}^{i}(U)\right)$ is connected whenever it is the support of $\mathcal{I}_{\beta_{i}+1-j}\left(\phi_{i}\right)$, or more generally, $\mathbb{P}\left(R_{j}^{i}(U)\right)$ is 
connected away from the components of $\mathbb{P}\left(R_{1}^{i-1}(U)\right)$ which are disconnected from the support of $\mathcal{I}_{\beta_{i}+1-j}\left(\phi_{i}\right)$.

\section{Acknowledgment}

I would like to thank Botong Wang for very useful discussions.

\section{References}

[1] N. Budur, Complements and higher resonance varieties of hyperplane arrangements, Math. Res. Lett. 18(5) (2011), 859-873.

Department of Mathematics, University of Notre Dame, 255 Hurley Hall, Notre Dame, IN 46556, USA

E-mail address: nbudur@nd.edu 